\title{
RELAÇÃO ENTRE BISFOSFONATO E PERIIMPLANTITE: Uma revisão sistemática de literatura
}

DOI: $10.22289 / 2446-922 X . V 3 N 1 A 1$

\author{
Diogo Alves de Araújo ${ }^{1}$ \\ Joice Silva Couto \\ Lilian de Barros
}

\section{RESUMO}

Bisfosfonatos são potentes inibidores usados pela medicina contra reabsorções ósseas, que tem sido considerada a melhor escolha terapêutica em doenças que afetam o metabolismo ósseo. O objetivo deste estudo é analisar a relação entre a terapia com bisfosfonatos e a periimplantite em pacientes reabilitados com implantes. O método empregado no estudo foi uma revisão sistemática de base qualitativa do tipo analítica. Para a pesquisa bibliográfica, utilizou-se a base de dados (PubMed - Biblioteca nacional de Medicina US) realizada no período compreendido de agosto de 1966 à agosto de 2016, sendo selecionados 28 artigos. $\mathrm{Na}$ análise de dados, foram encontrados 8 artigos que evidenciaram diagnóstico de osteonecrose associada aos bisfosfonatos da mandíbula, exposição óssea necrótica intraoral, número de implantes e acompanhamento, mostrando algumas variáveis, como ausência de evidência de perda óssea ou periimplantite com osteonecrose ao redor dos implantes dentários. Os resultados da pesquisa apontam que não só a intervenção cirúrgica de um implante dentário, mas o próprio implante, parece ser um fator de risco contínuo para o desenvolvimento da osteopatologia. Com a administração oral, após algumas semanas de ingestão, níveis adequados de inibição da reabsorção óssea podem ser observados. De acordo com vários estudos, os procedimentos odontológicos são um dos fatores de risco para o desenvolvimento da osteonecrose da mandíbula associada aos bisfosfonatos. Portanto, o acompanhamento cuidadoso e frequente de pacientes com implantes dentários recebendo terapia de bisfosfonato torna-se indicado.

Palavras-Chave: Peri-implantite, bisfosfonato, implantes dentais

\section{ABSTRACT}

\footnotetext{
${ }^{1}$ Endereço eletrônico de contato: diogoalves_15@yahoo.com.br
}

Recebido em 06/01/2017. Aprovado pelo Conselho Editorial e aceito para publicação em 18/04/2017.

Rev. Psicol Saúde e Debate. Jul., 2017:3(1):1-11. 
Bisphosphonates are bone resorption and potent osteoclast inhibitors medicine, which has been considered the best choice therapy in diseases affecting bone metabolism. The aim of this study is analyze the relationship between bisphosphonate therapy and peri-implantitis in patients who were rehabilitated with implants. The methodology used in this study was a qualitative systematic review of the analytical type. For the bibliographic research, the database (PubMed - National Library of Medicine US) was used between August of 1966 and August of 2016, being selected 28 articles. In the data analysis, eight articles were found that showed a diagnosis of osteonecrosis mandibular associated with bisphosphonates, intraoral necrotic bone exposure, number of implants and follow-up, showing some variables such as absence of evidence of bone loss or peri-implantitis with osteonecrosis around implants dental procedures. The results of the research indicate that not only the surgical intervention of a dental implant, but the implant itself, seems to be a continuous risk factor for the development of osteopathology. With oral administration, after a few weeks of ingestion, adequate levels of inhibition of bone resorption may be observed. According to several studies, dental procedures are one of the risk factors for the development of osteonecrosis of the mandible associated with bisphosphonates. Therefore, careful and frequent follow-up of patients with dental implants receiving bisphosphonate therapy is advised.

Keywords: peri-implantitis, bisphosphonate, dental implants

\section{INTRODUÇÃO}

Bisfosfonatos são potentes inibidores usados pela medicina contra reabsorções ósseas, que tem sido considerada a melhor escolha terapêutica em doenças que afetam o metabolismo ósseo. Eles foram utilizados para doenças como osteoporose, doença de Paget e tumores malignos, como mieloma múltiplo, hipercalcemia e outros com metástase óssea como próstata e câncer de mama. ${ }^{(1,20)}$

Existem duas categorias principais dos bisfosfonatos, os bisfosfonatos não contendo nitrogênio e os bisfosfonatos contendo nitrogênio. ${ }^{(2)} E$ a adição de um grupo amina ao fim de uma cadeia que aumenta a potência do fármaco. ${ }^{(3,4)}$

Os bisfosfonatos podem ser administradas por via oral ou intravenosa - Tabela 1. Com a administração oral, mesmo após algumas semanas de ingestão, podem ser observados níveis adequados de inibição da reabsorção óssea. Estas dosagens são, no entanto, insuficientes para contrabalancear as atividades de reabsorção de tumores metastastáticos ósseos ou doença de Paget e nestas situações devem ser administradas intravenosos, onde o processo de alta reabsorção óssea pode ser interrompido em 24-48 horas. ${ }^{(5,6)}$

Rev. Psicol Saúde e Debate. Jul., 2017:3(1):1-11. 
Tabela 1 - Diferentes tipos e marcas comerciais de bisfosfonatos disponíveis.

\begin{tabular}{|c|c|c|c|c|}
\hline $\begin{array}{l}\text { Nome } \\
\text { Genérico }\end{array}$ & Marca & $\begin{array}{l}\text { Nome } \\
\text { Comercial }\end{array}$ & $\begin{array}{l}\text { Ingrediente } \\
\text { ativo }\end{array}$ & Administração \\
\hline $\begin{array}{l}\text { Acido } \\
\text { Zoledrônico }\end{array}$ & Zometa & $\begin{array}{l}\text { Zometa } \\
\text { Aclasta }\end{array}$ & Zoledronato & Via intravenosa \\
\hline Pamidronato & Aredia & $\begin{array}{l}\text { Aredia, } \\
\text { Linoten, } \\
\text { Pamifos, } \\
\text { Xinsidona }\end{array}$ & Pamidronato & Via intravenosa \\
\hline Etidronato & Didronel & $\begin{array}{l}\text { Osteum, } \\
\text { Difosfen }\end{array}$ & Etidronato & $\begin{array}{l}\text { Via oral }{ }^{*} / \\
\text { intravenosa }\end{array}$ \\
\hline Clodronato & Bonefos & Bonefos & Clodronato & $\begin{array}{l}\text { Via oral/ } \\
\text { intravenosa }\end{array}$ \\
\hline Ibandronato & $\begin{array}{l}\text { Bondronat or } \\
\text { Boniva }\end{array}$ & Bondronat & Ibandronato & $\begin{array}{l}\text { Via oral/ } \\
\text { intravenosa }\end{array}$ \\
\hline Tiludronato & Skelid & Skelid & Tiludronato & Via oral \\
\hline Risedronato & Actonel & Actonel, Acrel & Risedronato & Via oral \\
\hline Aledronato & Fosamax & $\begin{array}{l}\text { Fosamax, } \\
\text { Fosavance }\end{array}$ & Alendronato & Via oral \\
\hline
\end{tabular}

Os procedimentos odontológicos são um dos fatores de risco para o desenvolvimento da osteonecrose associada aos bisfosfonatos da mandíbula ${ }^{(7)}$.

Foi demonstrado que o principal gatilho de osteonecrose associada aos bisfosfonatos em pacientes tratados, foi a extração dentária ${ }^{(8)}$. Existem também diversos estudos que identificaram extrações dentárias ou procedimentos cirúrgicos invasivos como sendo o principal fator para o desenvolvimento de osteonecrose associada aos bisfosfonatos em mandíbula. ${ }^{(9,10,11,12)}$

Outros fatores também desempenham um papel importante no desenvolvimento da osteonecrose associada aos bisfosfonatos em pacientes que utilizam o medicamento via oral. ${ }^{(13)}$

É importante avaliar os fatores que colocam os pacientes em condições de risco para o desenvolvimento da osteonecrose associada aos bisfosfonatos, alguns deles são: a presença de tumor ósseo, a anemia, que afeta a capacidade de transporte de oxigênio, a perda de peso afetando a nutrição, o tabagismo, comprometimentos pulmonares, quimioterapia, radiação, esteróides e aumento da carga metabólica. ${ }^{(14)}$ Qualquer doença que afeta o metabolismo celular, a resposta imune e a oxigenação, pode funcionar como importante fator para o desenvolvimento de osteonecrose associada aos bisfosfonatos. $\mathrm{A}$ obesidade também é conhecida como um fator na osteomielite dos ossos longos e mandíbula e pode ser um fator de osteonecrose associada aos bisfosfonatos também. ${ }^{(13)}$ 
O primeiro caso de osteonecrose da mandíbula envolvendo um paciente tratado com bisfosfonato foi relatado por Marx ${ }^{15}$ em $2003^{(15,16)}$ e observou-se que a freqüência de osteonecrose da mandíbula é maior nos pacientes que receberam a forma intravenosa de bisfosfonato. ${ }^{(15,17)}$ No entanto, foi publicado o primeiro relato da influência do bisfosfonato no tratamento com implantes dentários, descrevendo um paciente que tinha cinco implantes instalados na região inferior anterior com osseointegração bem sucedida, e em seguida, tomou etidronato dissódico por via oral para osteoporose, resultando no deslocamento de todos os cinco implantes após 5 meses. ${ }^{(18)}$

Os efeitos a curto e longo prazo dos bifosfonatos na osseointegração dos implantes dentários são ainda controversos, no entanto, alguns estudos mostraram para aqueles pacientes que receberam bisfosfonatos mais potentes, apresentaram falha do implante e a cicatrização tardia do sítio cirúrgico. ${ }^{(19)}$

Uma anamnese detalhada e um exame oral completo devem ser realizados antes do tratamento com bifosfonatos, bem como quaisquer dentes condenados devem ser extraídos, finalizar todos os procedimentos odontológicos invasivos e buscar uma condição periodontal ideal. Como implantes dentários e terapias com bisfosfonato são tratamentos muito comuns em pacientes adultos, há uma grande preocupação com as consequências da reabilitação com implantes dentários em pacientes que fazem uso do medicamento principalmente relacionados com os riscos envolvidos no desenvolvimento da osteonecrose da mandíbula.

Por consequência, o objetivo desta revisão sistemática de literatura é analisar a relação entre a terapia com bisfosfonatos e periimplantite em pacientes que foram reabilitados com implantes.

\section{METODOLOGIA}

Uma pesquisa eletrônica utilizando banco de dados do PubMed foi realizada no período de agosto de 1966 à agosto de 2016 buscando artigos publicados na literatura odontológica com as seguintes palavras-chave: "periimplantitis" e "bisphosphate".

$\mathrm{Na}$ análise de dados, foram encontrados 28 artigos, destes, 8 foram evidenciados. Os critérios de inclusão deveriam apresentar os seguintes temas, como: ausência de evidência de perda óssea ou periimplantite com osteonecrose circundando os implantes dentários, diagnóstico de osteonecrose da mandíbula associada ao uso do bisfosfonato, exposição óssea necrótica intraoral, número de implantes e acompanhamento. Estes dados foram coletados e apresentados na (TABELA 2), de acordo com cada autor. Os critérios de exclusão foram definidos da seguinte forma: não serem publicados na língua inglesa e não 
terem correlação entre sinais ou sintomas de periimplantite associada ao bisfosfonato (TABELA 3).

Tabela 2: Estudos incluídos

\begin{tabular}{|c|c|c|c|c|}
\hline Autores & $\begin{array}{l}\text { Tipo de } \\
\text { Estudo }\end{array}$ & $\begin{array}{l}\text { Numero de } \\
\text { Implantes }\end{array}$ & $\begin{array}{ll}\text { Tempo } & \text { de } \\
\text { acompanhamento } & \end{array}$ & Medicamento \\
\hline $\begin{array}{l}\text { Kwon et al; } \\
2014 .\end{array}$ & Estudo Clínico & - & +-30 meses & Alendronato \\
\hline $\begin{array}{l}\text { Yuan et al; } \\
2012\end{array}$ & $\begin{array}{l}\text { Relato de } \\
\text { Caso }\end{array}$ & 2 & $\begin{array}{c}1 \text { ano } \\
3 \text { anos } \\
7 \text { anos }\end{array}$ & $\begin{array}{c}\text { Risendronato } \\
\text { Alendronato } \\
\text { - }\end{array}$ \\
\hline $\begin{array}{l}\text { Bell et al; } \\
2011\end{array}$ & $\begin{array}{c}\text { Estudo } \\
\text { Retrospectivo }\end{array}$ & 24 & +- 19 meses & - \\
\hline $\begin{array}{l}\text { Shabestari } \\
\text { et al; } 2009\end{array}$ & $\begin{array}{l}\text { Serie de } \\
\text { Casos }\end{array}$ & 46 & 4 anos & Alendronato \\
\hline $\begin{array}{l}\text { Shibutani } \\
\text { et al; } 2001\end{array}$ & $\begin{array}{c}\text { Estudo } \\
\text { Experimental }\end{array}$ & 20 & 12 semanas & Pamidronato \\
\hline
\end{tabular}

$(21,22,23,24,25)$

Tabela 3: Estudos excluídos

Autor Critério de exclusão do estudo

Borm et al; $2013 \quad$ Lingua alemã

Serino G, Turri A. Ausência de tratamento com bisfosfonato associado a 2011

Kivelä-Rajamäki et Ausência de tratamento com bisfosfonato associado a al; 2003 periimplantite

$(26,27,28)$

Rev. Psicol Saúde e Debate. Jul., 2017:3(1):1-11. 


\section{ANÁLISE DE DADOS}

Os artigos forma lidos de forma completa e os dados foram apresentados em forma de tabela de acordo com o autor, ano de publicação, tipo de estudo, número de implantes instalados, tempo de acompanhamento e medicamento utilizado.

\section{DISCUSSÃO}

Foi apresentado um estudo onde um implante dentário osseointegrado ocorreu osteonecrose ao redor do implante após a administração do bisfosfonato. Neste estudo prospectivo, alguns pacientes com osteonecrose ao redor de implantes foram recrutados e tratados com bifosfonato. A maioria tinha sido tratada para osteoporose e apenas um para 0 mieloma múltiplo. A medicação foi interrompida e uma cirurgia foi realizada sobre aqueles que não apresentaram melhora. Foram realizadas biópsia e análise da peça cirúrgica bem como análise histológica e histomorfométrica. O estudo envolveu a avaliação de 19 pacientes (masculino: feminino $=2: 17$ ). $O$ uso de bisfosfonato via oral foi dominante $(n=$ $15,79 \%$ ) em relação a intravenosa. O fármaco associado mais comum foi o Alendronato. Os pacientes foram divididos em dois grupos de acordo com o tempo de início da administração: antes da iniciação terapia medicamentosa $(n=16)$ e após a cirurgia de implante $(n=3)$. Apenas três pacientes desenvolveram osteonecrose nos 6 meses após a cirurgia de implante. Nove pacientes mostraram sucesso da osseointegração após a instalação de implantes em um período médio de 35 meses até o desenvolvimento de osteonecrose. O tratamento com bisfosfonato iniciado em pacientes após cirurgia de implante mostrou que dois pacientes mantiveram seus implantes com sucesso por mais de dois anos. A osteonecrose associado ao bisfosfonato na mandíbula foi desencadeada após a administração do bisfosfonato e não pela cirurgia. Um paciente desenvolveu osteonecrose na mandibula após 18 meses de controle com bisfosfonato. O resultado mostrou que a maioria dos pacientes ( $n=11 / 19,58 \%$ ) neste estudo desenvolveu osteonecrose associada ao bisfosfonato não apresentando relação ao trauma cirúrgico de inserção ou remoção do implante dentário. ${ }^{(21)}$

Foi relatado um caso, em que os implantes infectados do paciente tinham sido colocados 7 anos antes do início do tratamento com bisfosfonato. O paciente teve dois implantes colocados na região do $1^{\circ}$ e do $2^{\circ}$ molar inferior esquerdo. $O$ paciente foi diagnosticado com osteoporose e começou a tomar Residronato, um ano depois, a droga foi alterada para Alendronato. Três anos após o início do tratamento com bisfosfonato, o 
paciente começou a apresentar sintomas de infecção periimplantar, o que de fato foi diagnosticado como osteonecrose da mandíbula associada ao bisfosfonato. ${ }^{(22)}$

Num estudo no qual os implantes foram instalados em locais logo após extrações dentárias devido patologias periapicais, foram avaliados com inúmeras variáveis. Dos 922 implantes analisados, um total de 285 implantes foram colocado em locais com radiolucências periapicais, sendo considerado como o grupo controle. Por outro lado, 637 implantes foram colocados em locais sem radiolucências periapicais. Além disso, neste estudo, vinte e quatro dos implantes foram colocados em pacientes tomando bisfosfonatos, dos quais, seis foram colocados em locais com radiolucências periapicais. Nenhum implante falhou nos pacientes que faziam uso da terapia com bifosfonatos. No entanto, 0 acompanhamento médio dos pacientes foi de aproximadamente 19 meses, o máximo de 93 meses e mínimo de 3 meses, ele não apontanto qualquer grupo e seu acompanhamento com clareza. ${ }^{(23)}$

Outro estudo mostrou que o tratamento com implante poderia ser diferente em pacientes que tomavam bifosfonatos. Em um estudo retrospectivo, verificou-se que 14 pacientes iniciaram a terapia com bisfosfonato após a instalação do implante e 7 pacientes estavam em tratamento com bifosfonato antes da colocação do implante. Os resultados do estudo mostraram que os 21 pacientes não apresentaram achados clínicos e radiológicos patológicos nos implantes. Também foi relatado que mesmo após 4 anos de tratamento com bisfosfonato não resultou em osteonecrose das mandíbulas após a instalação dos implantes. Além da terapia com bisfosfonato, todos os pacientes receberam cálcio e vitamina $D$ para tratar a osteoporose. Os pacientes receberam um ou mais implantes cerca de 4 anos antes do delineamento do estudo. Todos os implantes foram carregados cerca de 3 a 5 meses após a instalação. Os pontos clínicos avaliados incluem sangramento na sondagem, profundidade de bolsa, mobilidade e exposição tecidual. Estes foram relatados ao longo do estudo com uma avaliação da higiene oral convencional e individualizada de cada paciente. Uma bolsa periimplantar foi encontrada em quatro locais por implante (mesial, distal, bucal e lingual). As pontuações de profundidade de bolsa foram registadas em (presente $=1$, ausente $=0$ ). As alterações no osso marginal foram avaliadas pela medição da exposição tecidual utilizando radiografias periapicais. Nenhum dos implantes mostrou mobilidade. Todos esses pacientes apresentaram livres de peri-implantite. O tempo de tratamento com bifosfonato, antes e após a inserção do implante não mostrou influência estatisticamente significativa na profundidade de bolsa, sangramento a sondagem e mobilidade, da mesma forma, a localização do implante, tipo de prótese e antagonista 
nãoapresentaram influência significativa no implante para parâmetros clínicos e radiológicos. (24)

A inibição da reabsorção óssea alveolar em periodontite foi relatada em um estudo experimental mostrando resultados que sugeriram que Pamidronato pode inibir a progressão da reabsorção óssea alveolar em cães. O Pamidronato pode prevenir a reabsorção óssea alveolar na periimplantite e pode produzir um resultado mais previsível no tratamento de implantes dentários. Um total de 10 cães da raça Beagle foram usados para o estudo. Os $2^{\circ}$ e 3ํmolares inferiores esquerdos dos cães foram extraídos e após 6 meses, um conjunto de dois implantes dentários foram colocados. Os pilares de cicatrização foram colocados 4,5 meses após os implantes. A osteointegração foi confirmada em todos os casos. Os implantes dentários não foram colocados durante o tratamento. Um dispositivo de "seda" foi instalado em torno do pilar de cicatrização. Um grupo experimental de 5 cães foram submetidos ao uso do Pamidronato via intramuscular a cada 3 dias. O restante dos cães fizeram parte do grupo controle. Amostras de sangue e urina foram coletadas todas as semanas e também radiografias para avaliar a crista óssea alveolar. A superfície do implante foi coberta com placas e após 12 semanas houve uma evidente inflamação gengival em torno dos implantes. Foi feita uma medida da distância entre a superfície superior e o fundo do defeito criado. A distância foi significativamente menor no grupo tratado com Pamidronato. ${ }^{(25)}$ Embora, o risco de desenvolver osteonecrose de mandibula usando bifosfonatos orais pareça menor, o uso do medicamento com duração maior que três anos deve ser cautelosamente observado. Este tempo pode ser reduzido na presença de algumas comorbidades, como o uso de corticosteróides crônicos. Pode ser considerada pelo clínico a descontinuação de bisfosfonatos por um período de três meses antes e após a cirurgia dentária invasiva, diminuindo o risco de osteonecrose. A justificativa para esta abordagem é baseada nas instabilidades da função osteoclástica demonstrada em dados que estão ligados à terapia com bifosfonato e estudos recentes mostram um melhor resultado do tratamento com a interrupção do medicamento. ${ }^{(20)}$

Encontramos nesta revisão de literatura que o uso do bisfosfonato é um assunto controverso necissitando estudos mais aprofundados visando esclarecer seu uso relacionado com tratamentos dentários. A incidência de osteonecrose oral em pacientes com história de tratamento com bifosfonatos intravenosos tem sido relatada e associada mais comumente à extração dentária. Preocupações com osteonecrose da mandíbula e a falha do implante representam dois desfechos muito distintos que precisam ser considerados individualmente e estudos futuros devem ser realizados para um melhor planejamento do tratamento odontológico.

Rev. Psicol Saúde e Debate. Jul., 2017:3(1):1-11. 


\section{CONSIDERAÇÕES}

Diversas conclusões podem ser consideradas nesta revisão de literatura. Implantes dentários e bisfosfonatos estão relacionados à osteonecrose da mandíbula em pacientes que receberam tratamentos para muitas doenças do metabolismo ósseo, especialmente para a osteoporose. A instalação do implante na região posterior da mandíbula parece aumentar o risco de desenvolver osteonecrose da mandíbula associado ao bisfosfonato. Como a maioria dos casos relatados de osteonecrose na mandíbula e implantes dentários ocorrem na região posterior, alguns fatores devem ser cuidadosamente considerados antes da instalação do implante em pacientes que recebem tratamento com bisfosfonato a longo prazo. Não só a intervenção cirúrgica de um implante dentário, mas também o próprio implante parece ser um fator de risco contínuo no desenvolvimento da osteonecrose. Cabe ao cirurgião dentista, intervir, suspendendo o uso do medicamento para tratamento odontológico se necessário, assim como cuidado e acompanhamento do paciente.

\section{REFERÊNCIAS}

1 - Madrid C, Sanz M. Qual o impacto dos bisfosfonatos administrados sistematicamente na terapia de implante oral? Uma revisão sistemática. Clin Implantes orais Res. 2009 Sep; 20 Suppl 4: 87-95.

2 - Green J. Bisfosfonatos: Revisão pré-clínica. J Oncologist 2004; 9: 3-13.

3 - Fleisch H, Russell RG, Straumann F. Efeito do pirofosfato na hidroxiapatita e suas implicações na homeostase do cálcio. Natureza. 1966 Nov 26; 212 (5065): 901-3.

4 - Memon S, Weltman RL, Katancik JA. Bifosfonatos orais: sucesso precoce do implante dentário endóseo e alterações ósseas crestais. Estudo retrospectivo. Int J Oral Maxillofac Implantes. 2012 Set-Out; 27 (5): 1216-22.

5 - Wang HL, Weber D, McCauley LK. Efeito de bisfosfonatos orais a longo prazo na cicatrização de feridas de implantes: revisão da literatura e relato de um caso. J Periodonto. 2007 Mar; 78 (3): 584-94. Revisão.

6 - Montoya-Carralero JM, Parra-Mino P, Ramírez-Fernández P, Morata-Murcia IM, Mompeán-Gambin mdel C, Calvo-Guirado JL. Implantes dentários em pacientes tratados com bifosfonatos orais: uma revisão bibliográfica. Med Oral Patol Oral Cir Bucal. 2010 Jan 1; 15 (1): e65-9. Revisão.

7 - Moinzadeh AT, Shemesh H., Neirynck NA, Aubert C, Wesselink PR. Bisfosfonatos e suas implicações clínicas na terapia endodôntica. Int Endod J. 2013 May; 46 (5): 391-8. 
8 - Mavrokokki T, Cheng A, Stein B, Goss A. Natureza e frequência da osteonecrose associada aos bisfosfonatos das mandíbulas na Austrália. J Oral Maxillofac Surg. 2007; 65: 415-423.

9 - Marx RE, Sawatari Y, Fortin M, Broumand V. O osso exposto induzido por bisfosfonatos (osteonecrose / osteopetrose) dos maxilares: fatores de risco, reconhecimento, prevenção e tratamento. J Oral Maxillofac Surg. 2005; 63: 1567-1575.

10 - Pazianas M, Miller P, Blumentals WA, Bernal M, Kothawala P. Revista da literatura sobre osteonecrose da mandíbula em pacientes com osteoporose tratados com bifosfonatos orais: prevalência, fatores de risco e características clínicas. Clin Ther. 2007; 29: 1548-1558.

11 - Hoff $\mathrm{AO}$, Toth $\mathrm{BB}$, Altundag $\mathrm{K}$, et ai. A freqüência e os fatores de risco associados à osteonecrose da mandíbula em pacientes com câncer tratados com bifosfonatos intravenosos. J Bone Miner Res 2008; 6: 826.

12 - Filleul O, Crompot E, Saussez S. Osteonecrose induzida por bisfosfonatos da mandíbula: revisão de 2.400 casos de pacientes. J Cancer Res Clin Oncol. 2010 Agosto; 136 (8): 1117-24.

13 - Wessel JH, Dodson TB, Zavras Al. Zoledronato, tabagismo e obesidade são fortes fatores de risco para osteonecrose da mandíbula: um estudo caso-controle. J Oral Maxillofac Surg 2008; 66: 625-31.

14 - Assael LA. Bisfosfonatos e saúde bucal: cartilha e uma atualização para o cirurgião praticante. Oral Maxillofac Surg Clin North Am. 2011 Aug; 23 (3): 443-53.

15 - Marx RE. Pamidronato (Aredia) e zoledronato (Zometa) induziram necrose avascular das mandíbulas: uma epidemia em crescimento. J Oral Maxillofac Surg. 2003 Sep; 61 (9): 1115-7.

16 - Wang, J., Goodger, N.M. \& Pogrel, M.A. Osteonecrose das mandíbulas associadas à quimioterapia do cancro. O Jornal de Cirurgia Oral e Maxilofacial. 2003

17 - Ruggiero SL, Mehrotra B, Rosenberg TJ, Engroff SL. Osteonecrose das mandíbulas associadas ao uso de bisfosfonatos: uma revisão de 63 casos.J Oral Maxillofac Surg. 2004; 62: $527-534$.

18 - Starck WJ, Epker BN. Falha de implantes dentários osseointegrados após a terapia com difosfonato para osteoporose: relato de um caso. Int J Oral Maxillofac Implantes. 1995; 10: 74-78.

19 - Woo SB, Hellstein JW, Kalmar JR: Revisão narrativa (corrigida): Bisfosfonatos e osteonecrose das mandíbulas. Ann Intern Med 144: 753, 2006.

20 - Ruggiero SL. Osteonecrose associada aos bisfosfonatos da mandíbula (BRONJ): descoberta inicial e posterior desenvolvimento. J Oral Maxillofac Surg. 2009 Maio; 67 (5 Supl): 13-8.

21 - Kwon TG, Lee CO, Parque JW, Choi SY, Rijal G, Shin HI. Osteonecrose associada a implantes dentários em pacientes submetidos a tratamento com bifosfonatos. Clin Implantes orais Res. 2014 May; 25 (5): 632-40. 
22 - Yuan K, Chen KC, Chan YJ, Tsai CC, Chen HH, Shih CC. Insuficiência de implantes dentários associados à infecção bacteriana e uso prolongado de bifosfonatos: relato de caso. Implante Dent. 2012 Feb; 21 (1): 3-7.

23 - Bell CL, Diehl D, Bell, BM, Bell RE. Colocação imediata de implantes dentários em locais de extração com lesões periapicais: revisão retrospectiva de prontuários. J Oral Maxillofac Surg. 2011 Jun; 69 (6): 1623-7.

24 - Shabestari GO, Shayesteh YS, Khojasteh A, Alikhasi M, Moslemi N, Aminian A, Masaeli R, Eslami B, Treister NS. Colocação do implante em pacientes com tratamento com bifosfonatos por via oral: uma série de casos. Clin Implant Dent Relat Res. 2010 Sep; 12 (3): $175-80$.

25 - Shibutani T, Inuduka A, Horiki I, Luan Q, Iwayama Y. O bisfosfonato inibe a reabsorção óssea alveolar em perimplantites induzidas experimentalmente em cães. Clin Implantes orais Res. $2001 \mathrm{Abr} ; 12$ (2): 109-14.

26 - Borm JM, Moser S, Locher M, Damerau G, Stadlinger B, Grätz KW, Jacobsen C. [Avaliação do risco em pacientes submetidos a terapia anti-reabsorção óssea em odontologia. Uma atualização]. Schweiz Monatsschr Zahnmed. 2013; 123 (11): 985-1001; 955.Review. Francês, alemão.

27 - Serino G, Turri A. Extensão e localização da perda óssea em implantes dentários em pacientes com peri-implantite. J Biomech. 2011 Jan 11; 44 (2): 267-71.

28 - Kivelä-Rajamäki M, Maisi P, Srinivas R, Tervahartiala T, Teronen O, Husa V e Salo T, Sorsa T. Níveis e formas moleculares de MMP-7 (matrilisina 1) e MMP-8 (colagenase 2) Em fluido sulcular peri-implante humano doente. J Periodontal Res. 2003 Dec; 38 (6): 583-90. 